Trauma Berufskrankh $2005 \cdot 7[$ Suppl 1]:S231-S237 DOI 10.1007/s10039-004-0971-9

Online publiziert: 11. November 2004

๑) Springer Medizin Verlag 2004
D. Kügler · J. Barth · Medizinische Klinik, BG-Kliniken Bergmannstrost, Halle, Saale

\section{Komplikation Lungenembolie}

Fällen jährlich pulmonale Embolien als todesursächliches oder zumindest teilursächliches Ereignis anzuschuldigen sein [4].

Vor diesen Hintergrund wird deutlich, dass alle Anstrengungen unternommen werden müssen, um eine Lungenembolie zu verhindern. Dies erfordert einerseits prophylaktische Maßnahmen mit z. T. auch hohem finanziellem Aufwand zur Vermeidung von Thrombosen im venösen System, andererseits muss ein embolieverdächtiges klinisches Bild einer raschen und beweisenden Diagnostik zugeführt werden, damit unverzüglich eine effektive Therapie eingeleitet werden kann.

\section{Diagnostik}

Im Allgemeinen liegt das diagnostische Problem der akuten Lungenembolie nicht im Fehlen ausreichender Methoden, sondern in der Deutung der meist uncharakteristischen klinischen Symptome (• Tabelle 3) und Befunde (- Tabelle 4). Die weitere Diagnostik umfasst elektrokardiographische, röntgenologische und laborchemische Untersuchungen einschließlich Blutgasanalyse. Die transthorakale Echokardiographie, das Mehrschichtspiral-CT sowie farbduplex/dopplersonographische bzw. kompressionssonographische Untersuchungen sind
Über die Inzidenz der Erkrankung existieren in der Literatur keine einheitlichen Angaben. Nach Schätzungen und retrospektiven Analysen ist eine Lungenembolieereignisrate von etwa 6o-80 Fällen pro Jahr bezogen auf 100.000 Patienten anzunehmen [21]. Vermutlich liegt die Dunkelziffer weitaus höher, wenn diesbezüglich den Obduktionsstatistiken geglaubt werden kann [4].

Die Letalität der akuten Lungenembolie beträgt trotz zunehmender Therapieoptionen über 10\% [3] und ist abhängig vom

- Schweregrad des Ereignisses per se,

- vorbestehenden Begleiterkrankungen ( $\bullet$ Tabelle 1$)$ und Risikofaktoren (• Tabelle 2) sowie

- Zeitfenster des Erkennens der Lungenembolie bis zur Einleitung einer suffizienten Behandlung.

Dieses Zeitfenster kann sehr klein sein. Etwa 20\% der Patienten mit letztlich tödlicher Lungenembolie verstarben innerhalb von $2 \mathrm{~h} \mathrm{[9]}$.

Insgesamt - inklusive nicht postoperativer Lungenembolien - dürften in etwa 50.000

\section{Tabelle 1}

Prädisponierende Faktoren/Erkrankungen für thrombembolische Ereignisse

\begin{tabular}{ll}
\hline Angeborene Zustände & Erworbene Zustände \\
Mangel an Gerinnungsfaktoren & Exsikkose, Hämoglobinkonzentration \\
- Antithrombin III & Lupusantikoagulans \\
- Protein C & Antiphospholipidsyndrom \\
- Protein S & Neoplasma \\
• Heparinkofaktor II & Myeloproliferative Erkrankungen \\
\hline Mutationen von Gerinnungsfaktoren & Nephrotisches Syndrom \\
• Prothrombin & Leberzirrhose \\
- APC-Resistenz (Faktor-V-Leiden) & Östrogene, Schwangerschaft, Wochenbett \\
\hline $\begin{array}{l}\text { Störungen der Fibrinolyse } \\
\text { - Mangel an Plasminogen }\end{array}$ & Eklampsie, Präeklampsie \\
- Mangel an Gewebeplasminogenaktivator & Infektionskrankheiten, Sepsis \\
- Erhöhung des Gewebeplasminogenaktiva- & Chronisch-entzündliche Darmerkrankungen \\
torinhibitors & Chronisch-obstruktive Atemwegserkrankun- \\
• Hyper- oder Dysfibrinogenämie & gen (COPD) \\
\hline Andere Störungen & Morbus Behçet \\
• Erhöhte Konzentration des Faktors VIII & Paroxysmale nächtliche Hämoglobulinurie \\
- Sichelzellanämie & Hyperviskositätssyndrome \\
- Klinefelter-Syndrom & Apoplex, Immobilisation \\
• Hyperhomozysteinämie & Herzinsuffizienz, Adipositas \\
Modifiziert nach Heinrich [7] & Heparininduzierte Thrombozytopenie Typ II \\
\hline & \\
\hline
\end{tabular}


Tabelle 2

Risikofaktoren einer Lungenembolie

\begin{tabular}{lcc}
$\begin{array}{l}\text { Risikofaktoren } \\
{[\%]}\end{array}$ & $\begin{array}{l}\text { Alter } \\
<\mathbf{4 0} \text { Jahre }\end{array}$ & $\begin{array}{l}\text { Alter } \\
>\mathbf{4 0} \text { Jahre }\end{array}$ \\
$\begin{array}{l}\text { Trauma } \\
\text { Neoplasma }\end{array}$ & $\mathbf{2 8}$ & 3 \\
\hline Immobilisierung & 43 & 45 \\
\hline Längere Reisen & 10 & 3 \\
\hline $\begin{array}{l}\text { Frühere } \\
\text { Lungenembolien }\end{array}$ & 5 & 13 \\
\hline $\begin{array}{l}\text { Frühere Bein- } \\
\text { venenthrombose }\end{array}$ & 10 & 13 \\
\hline $\begin{array}{l}\text { Chirurgischer } \\
\text { Eingriff }\end{array}$ & 20 & 28 \\
\hline $\begin{array}{l}\text { Östrogene/orale } \\
\text { Antikonzeptiva }\end{array}$ & 5 & 5 \\
\hline $\begin{array}{l}\text { Keine } \\
\text { Risikofaktoren }\end{array}$ & 28 & 15 \\
\hline
\end{tabular}

Modifiziert nach Green et al. [6]

Tabelle 3

Symptomatik der akuten

Lungenembolie [1]

\begin{tabular}{ll} 
Symptom & Häufigkeit [\%] \\
Dyspnoe & 85 \\
\hline Thoraxschmerz & 88 \\
\hline Angst & 59 \\
\hline Husten & 53 \\
\hline Hämoptysen & 30 \\
\hline Schweißausbruch & 27 \\
\hline Synkope & 13 \\
\hline Herzrasen & 44 \\
\hline
\end{tabular}

Tabelle 4

Klinische Befunde der akuten

Lungenembolie [1]

\begin{tabular}{|ll}
\hline Befund & Häufigkeit [\%] \\
Tachypnoe & 92 \\
\hline Rasselgeräusche & 58 \\
\hline Betonter 2. Herzton & 53 \\
\hline Tachykardie & 44 \\
\hline Fieber $\left(>37,8^{\circ} \mathrm{C}\right)$ & 43 \\
\hline 3. Herzton & 34 \\
\hline Schweißausbruch & 36 \\
\hline Thrombophlebitis & 32 \\
\hline Ödeme & 24 \\
\hline Herzgeräusche & 23 \\
\hline Zyanose & 19 \\
\hline
\end{tabular}

Tabelle 5

Elektrokardiographische Befunde bei akuter Lungenembolie [22]

\begin{tabular}{|lc} 
Befund & Häufigkeit [\%] \\
Sinusrhythmus & 80 \\
\hline Rechtsschenkelblock & 8 \\
\hline T-Inversion & 40 \\
\hline ST-Senkung & 25 \\
\hline Rhythmusstörung & 25 \\
\hline Leitungsstörung & 10 \\
\hline QRS-Achsendrehung & 15
\end{tabular}

Tabelle 6

Röntgenologische Befunde der akuten Lungenembolie [14]

\begin{tabular}{ll} 
Befund & $\begin{array}{l}\text { Häufigkeit } \\
{[\%]}\end{array}$ \\
\hline Zwerchfellhochstand & $40-60$ \\
\hline Infiltrat & 40 \\
\hline Pleuraerguss & 30 \\
\hline Plumpe Pulmonalgefäße & 40 \\
\hline Atelektase & 20 \\
\hline Regionale Minderperfusion & 15 \\
\hline Herzgrößenänderung & 10 \\
\hline
\end{tabular}

einfache, nichtinvasive und kostengünstige Verfahren, die mit ausreichender Sicherheit die Verdachtsdiagnose Lungenembolie bestätigen können bzw. mit denen bei embolieverdächtiger Klinik eine Thrombose des Venensystems als Quellgebiet für Embolisationen erkannt werden kann.

\section{Klinische Symptome und Befunde}

Die Symptome einer Lungenembolie sind häufig unspezifisch (• Tabelle 3), besonders dann, wenn kardiopulmonale Begleiterkrankungen vorliegen. Nur $20 \%$ der Patienten weisen die klassische Trias aus Dyspnoe, Hämoptysen und pleuritischem Schmerz auf [20]. Tritt aber das Symptom Hämoptoe z. B. bei einem jungen, ansonsten gesunden postoperativen Patienten auf, der keinerlei Gründe für eine andere Genese bietet, ist das Bluthusten als ein „diagnostisches Geschenk“ zu bewerten. Auch wenn es bei den Symptomen Dyspnoe und Thoraxschmerz viele Ursachen geben mag, sollte beim Vorhandensein von Risikofaktoren, wie in $\bullet \mathbf{T a}$ - belle 2 aufgeführt, an eine Lungenembolie gedacht werden.

Die klinischen Untersuchungsbefunde sind ebenfalls unspezifisch, und kein Befund ist für sich pathognomonisch; sie können jedoch die Verdachtsdiagnose stützen. Von den in - Tabelle 4 genannten Befunden kommen Tachypnoe und Tachykardie am häufigsten vor [8].

\section{Laborbefunde/Blutgasanalyse}

\section{D-Dimer}

Die laborchemische Bestimmung dieses Fibrinogenabbauprodukts hat sich aufgrund des schnellen, standardisierten und reproduzierbaren Nachweisverfahrens in der initialen Diagnostik der Thrombembolie durchgesetzt und ist zudem in vielen Studien gut untersucht. Die Sensitivität wird mit 98\% oder höher angegeben [11].

Normale, mittels ELISA gemessene DDimer-Spiegel können diagnostisch als Ausschlusskriterium für eine Lungenembolie gewertet werden. Ein normaler DDimer-Wert macht die Thrombose und in deren Folge eine pulmonale Embolie sehr unwahrscheinlich. Allerdings ist die Spezifität niedrig, da alle Erkrankungen oder Zustände, die mit einer Mikrothrombenbildung und reaktiven Fibrinolyse einhergehen (z. B. nach chirurgischen Eingriffen, schwere Infektionen, maligne $\mathrm{Tu}$ moren), erhöhte D-Dimer-Spiegel zeigen [2]. Erfahrungsgemäß sollten bei postoperativen Patienten ein Anstieg der D-Dimere mehrere Tage nach dem chirurgischen Eingriff sowie sehr hohe D-Dimer-Spiegel nach Operationen Anlass zur Thrombemboliediagnostik geben.

\section{Arterielle Blutgasanalyse}

Sie wird oft im Rahmen der differenzialdiagnostischen Überlegungen eingesetzt. Bei einer Lungenembolie zeigt sie typischerweise die Kombination von Hypoxämie und Hypokapnie. Oft ist jedoch nur der $\mathrm{pCO}_{2}-$ Wert erniedrigt, was signalisiert, dass nur durch eine Hyperventilation ein noch ausreichend hoher $\mathrm{pO}_{2}$-Wert aufrechterhalten werden kann. Selbst ein Normalbefund schließt eine Lungenembolie nicht aus.

\section{Weitere Laboruntersuchungen}

Alle weiteren laborchemischen Marker (z. B. LDH-Erhöhung) sind unspezifisch 
und zur Diagnostik der Lungenembolie nicht geeignet, aber im späteren Verlauf von Bedeutung.

Die Labormedizin hat in den letzten Jahren hinsichtlich der Thrombophilie einen großen Beitrag geleistet. Neben lang bekannten Risikokonstellationen durch vermindertes AT III sind gegenwärtig eine Vielzahl weiterer genetisch determinierter Defekte beschrieben (z. B. APC-Resistenz, Protein C und S), die ein erhöhtes Thrombophilierisiko aufweisen (• Tabelle 1). Sie sind zwar in der Akutdiagnostik nicht nützlich, haben aber für spätere Folgeentscheidungen einen hohen Wert und sollten deshalb in Akutsituationen und vor Antikoagulation - wegen der VitaminK-Abhängigkeit wesentlicher Gerinnungsproteine - veranlasst werden. In der rechten Spalte von - Tabelle 1 sind dazu erworbene, überwiegend laborchemisch fassbare Störungen aufgelistet, die ein erhöhtes Thromboserisiko beinhalten.

\section{Elektrokardiogramm}

Die Ausprägung der pathologischen EKGZeichen ist vom Schweregrad der Lungenembolie abhängig. - Tabelle 5 gibt einen Überblick über gelegentlich anzutreffende Veränderungen des EKG. Auch bei dieser Untersuchung gibt es keine beweisenden Zeichen einer Lungenembolie. Ein Lagetypwechsel, z. B. vom Linkstyp eines präoperativen EKG zu einem Rechtstyp im Embolie-EKG, sollte jedoch an eine Lungenembolie denken lassen. Die im EKG auftretenden Veränderungen beruhen auf einer erhöhten Druckbelastung mit konsekutiver Ischämie im rechten Ventrikel. Die meisten Patienten zeigen nur eine Tachykardie mit kurzzeitigen Rhythmus- und unspezifischen Erregungsrückbildungsstörungen.

\section{Röntgenthorax}

Eine konventionelle Thoraxaufnahme (- Abb. 1) ist ebenfalls nur selten für die Sicherung der Diagnose geeignet ( $\bullet$ Tabelle 6). Gelegentlich finden sich direkte Zeichen einer Lungenembolie wie z. B. Gefäßabbrüche (Westermark-Zeichen) oder eine Dilatation der Pulmonalarterie, des rechten Ventrikels und Vorhofs sowie der V. cava superior und der V. azygos. Als indirekte Zeichen sind manchmal ein Zwerch-

Trauma Berufskrankh $2005 \cdot 7[$ Suppl 1]:S231-S237

DOI 10.1007/s10039-004-0971-9

c) Springer Medizin Verlag 2004

D. Kügler · J. Barth

\section{Komplikation Lungenembolie}

\section{Zusammenfassung}

Lungenembolien sind häufige und schwerwiegende Komplikationen einer Krankenhausbehandlung. Sie erfordern eine rasche und richtige Diagnostik, um die dem Schweregrad der Erkrankung entsprechende Behandlung unverzüglich einleiten zu können. Wegen der uncharakteristischen klinischen Befunde sollten zuerst schnell durchzuführende, nichtinvasive Untersuchungsmethoden wie Echokardiographie und Mehrschichtspiraltomographie Anwendung finden. Unter den laborchemischen Parametern hat der D-Dimer-Test hinsichtlich thrombembolischer Ereignis-

\section{Pulmonary embolism as a complication}

\section{Abstract}

Pulmonary embolism is a frequent and serious complication in surgical patients in hospital. Rapid and accurate diagnosis is essential to allow treatment appropriate to its severity without delay. As clinical signs and symptoms are noncharacteristic, rapidly performed, noninvasive procedures should first be carried out, such as echocardiography and spiral computer tomography, to verify the diagnosis. Among laboratory methods, the D-dimer test is the only one that has major value in supporting or excluding a thrombotic/thromboembolic event. If findings remain equivocal ventilation-perfusion scin- se eine große Bedeutung. Bei unklaren Befunden ist eine weiterführende, teilweise zeitaufwändigere Diagnostik (wie Ventilationsperfusionsszintigraphie und Pulmonalisangiographie) gerechtfertigt. Da eine akute Lungenembolie häufig als Komplikation einer tiefen Beinvenenthrombose auftritt, kommt der Thromboseprophylaxe eine besondere und wichtige Bedeutung zu.

\section{Schlüsselwörter}

Lungenembolie - Thrombose - Diagnostik . Prophylaxe tigraphy and pulmonary angiography are justified, but these are now regarded as second-line diagnostic procedures in acute embolism because of logistic problems (availability, transport of unstable patients). Since acute pulmonary embolism is frequently a complication of deep vein thrombosis in the leg, however, prophylaxis of venous thrombosis remains particularly important.

\section{Keywords}

Pulmonary embolism - Thrombosis .

Diagnosis · Prophylaxis 


\section{Intensivmedizin des Polytraumas}
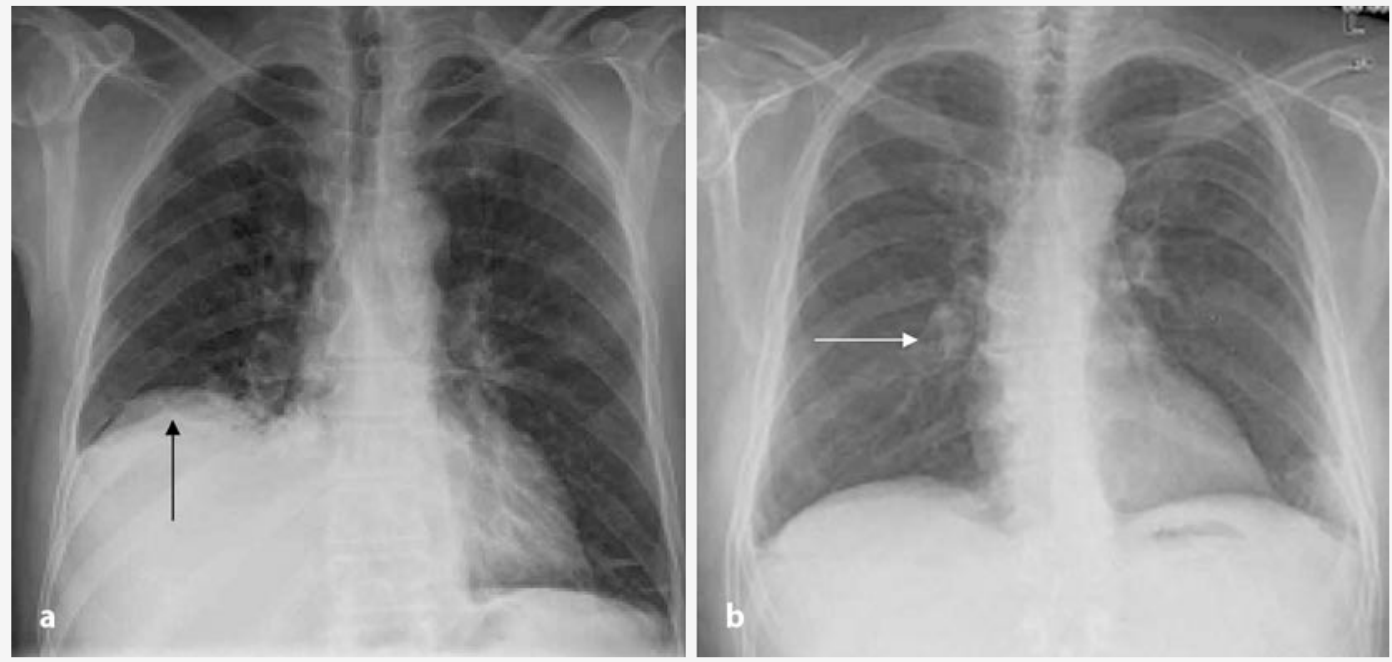

Abb. $1<$ Röntgenthoraxp.-a.-Aufnahme bei akuter Lungenembolie, a Zwerchfellhochstand, b „Hilusamputation" (Westermark-Zeichen)

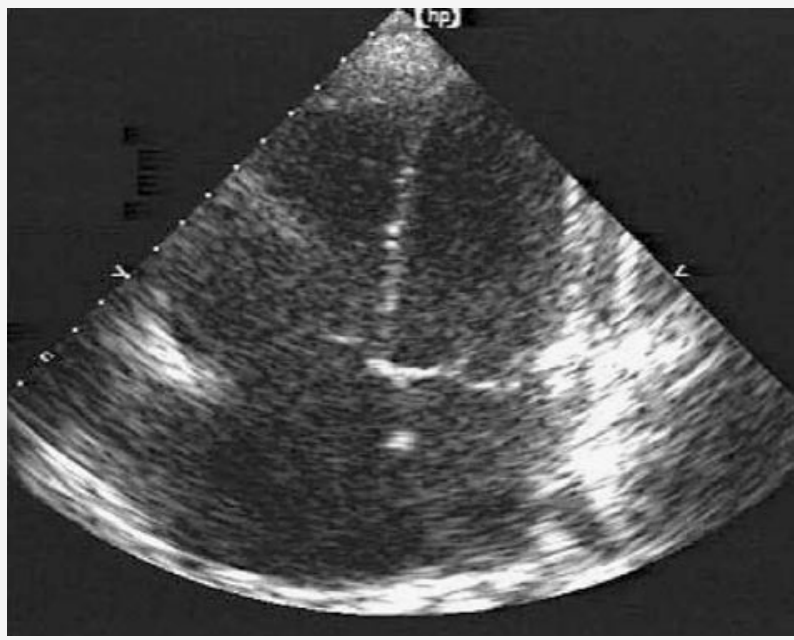

Abb. $2 \triangle$ Zweidimensionale Echokardiographie, akutes Cor pulmonale mit ausgeprägter rechtskardialer Dilatation ohne Nachweis einer rechtsventrikulären Hypertrophie



Abb. 3 Spiral-CT mit direkter Darstellung pulmonaler Embolien beidseits durch Kontrastmittelaussparung bzw. -abbruch fellhochstand, ein begleitender Pleuraerguss oder eine keilförmige Verschattung als Ausdruck des Perfusionsausfalls zu beobachten. Kleine Embolien können sich gelegentlich durch basal gelegene Plattenatelektasen (Fleischner-Linien) zu erkennen geben, die durch Verlust der Oberflächenspannung an den Alveolen entstehen [20].

\section{Transthorakale Echokardiographie}

Sie nimmt mit dem Nachweis einer rechtsventrikulären Dysfunktion eine zentrale Stellung in der Diagnostik der akuten Lungenembolie ein (• Abb. 2). Mit diesem bildgebenden Verfahren kann eine hämodynamisch relevante Embolie in kürzester Zeit am Patientenbett nachgewiesen oder ausgeschlossen werden (• Tabelle 7). Das
Erkennen einer hämodynamischen Beeinträchtigung stellt die Weichen für das weitere therapeutische Vorgehen (z. B. Lyse).

Die Echokardiographie ist auch bei $\mathrm{Pa}$ tienten mit fulminanter Lungenembolie und instabilen Kreislaufverhältnissen unverzüglich und direkt am Krankenbett möglich [12].

Die differenzialdiagnostisch zu beachtende chronische Rechtsherzbelastung geht im Gegensatz zur akuten Rechtsherzbelastung mit einer Hypertrophie des rechten Ventrikels einher. Weiterhin kann ein flottierender oder adhärenter Thrombus im rechten Herzen, $z$. B. bei einem postoperativen Patienten auf der Intensivstation mit liegendem zentralvenösem Venenkatheter direkt nachgewiesen werden. Dopplersonographisch ist zudem der pulmonalarterielle Druck direkt oder indirekt über die fast immer vorhandene Trikuspidalinsuffizienz messbar.

\section{Mehrschichtspiralcomputer- tomographie}

Sie hat sich aus röntgenologischer Sicht als die Methode der Wahl für die Diagnostik der zentralen Lungenembolie bis zur Segmentebene durchgesetzt (• Abb. 3, 4). Um die Pulmonalgefäße beurteilen zu können muss der Patient im Gegensatz zum MRT nur wenige Sekunden den Atem anhalten. Ein weiterer Vorteil ist die gleichzeitige Darstellung eines evtl. vorliegenden Lungeninfarkts oder einer Begleiterkrankung der Lunge, des Herzens und des Mediastinums. Bis zu einem gewissen Grad kann auch eine akute Embolie von einer älteren unterschieden werden [19]. 
Als sichere Zeichen einer Lungenembolie gelten Füllungsdefekte oder das so genannte Schienenstrangzeichen (umflossene lang gestreckte Aussparungen) und Wandunregelmäßigkeiten. Das Spiral-CT gilt deshalb als die sicherste und schnellste Methode, um eine zentrale - und damit massive oder submassive - Lungenembolie direkt nachzuweisen.

Des Weiteren kann der Kontrastmittelbolus bei schwer beeinträchtigten Patienten, da er für die Diagnostik ohnehin gegeben wird, zusätzlich für eine Abklärung des Venensystems des Abdomens und der unteren Extremitäten als häufigste Emboliequellen ausgenutzt werden [18]. Der CTBefund sagt jedoch nichts zur hämodynamischen Bedeutung des Embolieereignisses aus.

\section{Ultraschalluntersuchung des venösen Systems}

Die Kompressionssonographie als einfache und nichtinvasive Methode hat bei hoher Sensitivität und Spezifität aus internistischer Sicht bei Venenthrombosen als Emboliequelle einen hohen Stellenwert. Bei einer negativen Kompressionssonographie und einem negativen D-Dimer-Test ist auch eine akute Lungenembolie relativ unwahrscheinlich [5]. Bei freien Flussverhältnissen in den Venen, positivem D-DimerTest und embolieverdächtiger klinischer Situation ist eine Lungenembolie nicht ausgeschlossen, da gelegentlich auch das gesamte Material abgelöst worden sein kann.

Die Kompressionssonographie und farbkodierte Duplexsonographie mittels Dopplertechnik, bei denen sich zusätzlich Strömungen und Geschwindigkeiten des Bluts erfassen lassen, haben sich zudem als Standardmethoden zur Objektivierung einer tiefen Bein- oder Armthrombose etabliert [11].

\section{Erweiterte Diagnostik}

Bei stabilen Patienten und unsicheren Befunden können weitere Untersuchungen, die allerdings zeitaufwändiger, kostenintensiver und teils auch invasiver sind, zum Einsatz kommen. Weiterhin sollten Praktikabilitätsgründe und Verfügbarkeit diagnostischer Methoden Berücksichtigung finden.


Abb. $4<$ Spiral-CT, a Kontrastmittelabbruch als Zeichen eines embolischen Verschlusses in der rechten Pulmonalarterie und den Segmentarterien im rechten Ober- und Unterlappen, b sekundäre Rekonstruktion der pulmonalen Gefäßstruktur mit Kontrastmittelaussparung in 3D-Darstellung
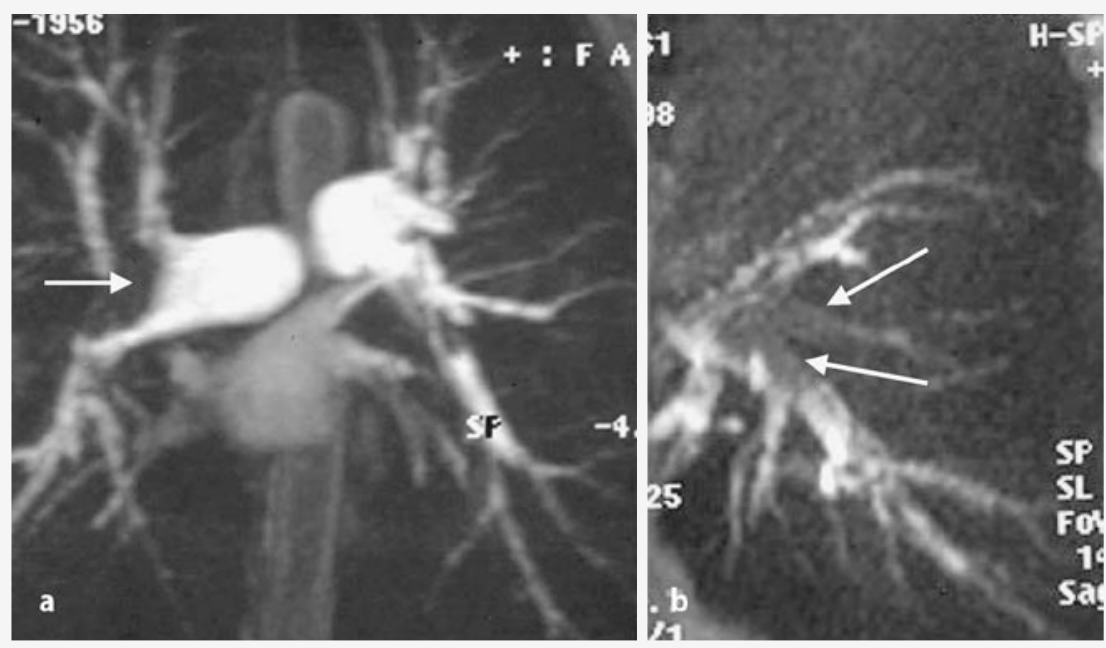

Abb. 5 MRT mit direkter Darstellung pulmonaler Embolien bis in die Peripherie, erkennbar an Kontrastmittelabbrüchen und -aussparungen, a thrombembolisches Material im Mittellappen, b multiple Embolien im linken Unterlappen

\section{Pulmonalisangiographie}

Sie galt viele Jahre als Goldstandard für die Objektivierung des Befundes einer Lungenembolie. Kritisch ist jedoch, dass einerseits die ständige Verfügbarkeit der Untersuchung, gerade als Notfalluntersuchung bei Nacht, nicht gegeben ist und andererseits die Methode aufgrund ihrer Invasivität gewisse Risiken für den Patienten aufweist.

Die Angiographie als ergänzende Untersuchung sollte genutzt werden, wenn bei fortbestehender Klinik die Diagnose z. B. mit der Echokardiographie und/oder Computertomographie nicht zu sichern war.

\section{Kombinierte Ventilations- und Perfusionsszintigraphie}

Mit ihr steht ein gutes bildgebendes Verfahren zum Ausschluss einer Lungenembolie zur Verfügung, weswegen diese Methode aus nuklearmedizinischer Sicht als Methode der Wahl bezeichnet wurde. Durch die 
Tabelle 7

Echokardiographische Befunde bei akuter und chronischer pulmonaler

Hypertonie

\begin{tabular}{ll}
$\begin{array}{l}\text { Akut } \\
\text { Rechtsventrikuläre Dilatation }>30 \mathrm{~mm}\end{array}$ & $\begin{array}{l}\text { Chronisch } \\
\text { Muskeldicke des rechten Ventrikels }>5 \mathrm{~mm}\end{array}$ \\
\hline $\begin{array}{l}\text { Maximale systolische Flussgeschwindigkeit } \\
\text { des Regurgitationsjets der } \\
\text { Trikuspidalinsuffizienz }>2,8 \mathrm{~m} / \mathrm{s}\end{array}$ & $\begin{array}{l}\text { Maximale systolische Flussgeschwindigkeit } \\
\text { des Regurgitationsjets der } \\
\text { Trikuspidalinsuffizienz }>3,8 \mathrm{~m} / \mathrm{s}\end{array}$ \\
\hline Hypokinesie des rechten Ventrikels & Normo-/Hyperkinesie des rechten Ventrikels \\
\hline Paradoxe Septumbewegung & \\
\hline $\begin{array}{l}\text { Diastolischer Diameter der Pulmonalarterie } \\
>12 \mathrm{~mm} / \mathrm{m}^{2}\end{array}$ & \\
\hline Modifiziert nach Hofmann et al. [10] & \\
\hline
\end{tabular}

Tabelle 8

Thromboserisikokategorien

\begin{tabular}{|c|c|c|c|}
\hline & Niedriges Risiko & Mittleres Risiko & Hohes Risiko \\
\hline $\begin{array}{l}\text { Chirurgische } \\
\text { Patienten }\end{array}$ & $\begin{array}{l}\text { Große Chirurgie, } \\
\text { Alter < } 40 \text { Jahre ohne } \\
\text { Risikofaktoren } \\
\text { Kleine Chirurgie, } \\
\text { Alter } 40-60 \text { Jahre } \\
\text { ohne Risikofaktoren, } \\
\text { kleinere Traumen }\end{array}$ & $\begin{array}{l}\text { Große Eingriffe, } \\
\text { Alter } 40-60 \text { Jahre } \\
\text { ohne Risikofaktoren } \\
\text { Kleinere Eingriffe, } \\
\text { Alter > } 60 \text { Jahre } \\
\text { Kleine Chirurgie im } \\
\text { Alter von } 40-60 \text { Jahren } \\
\text { mit früherer } \\
\text { Thrombembolie oder } \\
\text { Östrogentherapie }\end{array}$ & $\begin{array}{l}\text { Große Operationen, } \\
\text { Alter }>60 \text { Jahre } \\
\text { Große Eingriffe, } \\
\text { Alter } 40-60 \text { Jahre, } \\
\text { bei Malignomen oder } \\
\text { früherer Thrombembo- } \\
\text { lie } \\
\text { Fraktur oder ortho- } \\
\text { pädische Operation an } \\
\text { Becken, Hüfte, Beinen } \\
\text { Thrombophilie }\end{array}$ \\
\hline $\begin{array}{l}\text { Internistische } \\
\text { Patienten }\end{array}$ & $\begin{array}{l}\text { Leichtere internistische } \\
\text { Erkrankungen }\end{array}$ & $\begin{array}{l}\text { Immobilisierung, } \\
\text { kardiale Insuffizienz }\end{array}$ & $\begin{array}{l}\text { Apoplex } \\
\text { Alter }>70 \text { Jahre } \\
\text { Kardiale } \\
\text { Dekompensation } \\
\text { Frühere } \\
\text { Thrombembolie } \\
\text { Thrombophilie }\end{array}$ \\
\hline
\end{tabular}

geringe Verbreitung der kostenintensiven und strahlenbelastenden Untersuchung sollte die Szintigraphie nur in unklaren Fällen mit diskrepanten Befunden oder bei klinischem Verdacht auf chronisch rezidivierende Lungenembolien zur Anwendung kommen [15].

\section{Magnetresonanztomographie}

Sie ist hinsichtlich der diagnostischen Aussagekraft ungefähr mit dem Mehrschichtspiral-CT vergleichbar. Sie kann jedoch nicht bei schwer dyspnoischen Patienten eingesetzt werden, da diese kaum den Atem über die notwendige Untersuchungszeit von 20-30 s anhalten können. Subsegmentale Embolisationen können gelegent- lich weniger gut dargestellt werden [8]. Einen großen Vorteil bietet jedoch die Gabe eines paramagnetischen nichtjodhaltigen Kontrastmittels, sodass diese Untersuchung alternativ bei bekannten hyperthyreoten Patienten zum Einsatz kommen kann. Bei Ausdehnung der Untersuchung können analog dem Mehrschicht-SpiralCT Thrombosen in den Bein- und Beckenvenen dargestellt werden [13] (• Abb.5).

\section{Prophylaxe und Therapie}

Die Effizienz physikalischer und medikamentöser Maßnahmen zur Thromboseprophylaxe wurde in mehreren klinischen Studien nachgewiesen. Durch die Eintei- lung in niedrige, mittlere und hohe Risikogruppen (• Tabelle 8) und der damit verbundenen, der Kategorie entsprechenden Behandlung der Patienten konnte eine deutliche Reduktion von thrombembolischen Ereignissen um bis zu 50\% erreicht werden [17]. Allerdings haben diese Maßnahmen auch ihren Preis. So wurden in unserem Klinikum im Zeitraum von Januar bis Juni 2003 allein für die Thromboseprophylaxe mit niedermolekularen Heparinen fast 150.000 EUR ausgegeben.

Die medikamentöse Prophylaxe orientiert sich an den Risikogruppen. Die Gabe von unfraktionierten Heparinen ist weitgehend wegen geringerer Effektivität und den häufigeren Injektionen verlassen worden. Niedermolekulare Heparine können in einer Dosierung von 1750-5000 Anti-FXa-Einheiten 1- bis 2-mal täglich gegeben werden. Bei Hochrisikopatienten und ungenügender Mobilität ist eine Prophylaxedauer von mindestens 3 Wochen oder länger nach Krankenhausentlassung oder bis zur Gipsabnahme angezeigt [16].

$\mathrm{Zu}$ den etablierten physikalischen Methoden zählen die am häufigsten verwendeten Thromboseprophylaxestrümpfe (die ohnehin bei allen Risikogruppen als Basismaßnahme angewandt werden sollten), die intermittierende pneumatische Kompression sowie die Atemtherapie und die Krankengymnastik mit dem Ziel der Frühmobilisierung. Die Implantation eines so genannten Kavaschirms (V.-cava-Block) wird nur in Einzelfällen bei Kontraindikationen gegen eine antithrombotische Therapie eingesetzt.

Die Behandlung einer Lungenembolie selbst richtet sich nach dem Schweregrad und reicht von der konservativen Therapie mit unfraktioniertem PTT-gesteuertem oder niedermolekularem Heparin über Thrombolyse, meist mittels tPA oder Urokinase, bis hin zur chirurgischen Intervention. Des Weiteren ist eine effektive orale Antikoagulation mit einem Vitamin-KAntagonisten für mindestens 6 Monate erforderlich. Sollte es zu einer Rezidivembolie kommen, muss bei Nachweis eines hereditären Gerinnungsdefekts eine lebenslange Antikoagulation erfolgen [8].

\section{Fazit für die Praxis}

Für die Verdachtsdiagnose Lungenembolie gibt es keine typische Symptomatik 
und keine charakteristischen klinischen Befunde. Die elektrokardiographischen und röntgenologischen Befunde spielen überwiegend differenzialdiagnostisch eine wichtige Rolle. Mit dem laborchemischen D-Dimer-Test kann eine relevante Lungenembolie weitestgehend ausgeschlossen werden. Fällt dieser Test positiv aus, müssen bei entsprechender Klinik nichtinvasive Untersuchungsmethoden angewendet werden, wobei die transthorakale Echokardiographie einen wesentlichen Platz für die Erkennung eines akuten Cor pulmonale einnimmt. Wegen der hohen Wahrscheinlichkeit einer gleichzeitig vorliegenden Venenthrombose kann eine Kompressions- oder/und farbkodierte Duplexsonographie die Verdachtsdiagnose erheblich stützen. Bei einer fulminanten Lungenembolie ist jedoch der echokardiographische Befund so typisch, dass unverzüglich unter Verzicht weiterer Untersuchungen mit der Therapie begonnen werden kann. Das Mehrschichtspiral-CT hat sich wegen der ständigen Verfügbarkeit sowie der schnellen und sicheren Befunderhebung durchgesetzt.

Die Therapie der Lungenembolie richtet sich nach dem Schweregrad.

\section{Korrespondierender Autor Dr. D. Kügler}

Medizinische Klinik, BG-Kliniken Bergmannstrost, Merseburger Straße 165, 06112 Halle, Saale E-Mail: daniel.kuegler@bergmannstrost.com

\section{Danksagung}

Die röntgenologischen, computer- und magnetresonanztomographischen Aufnahmen wurden uns freundlicherweise von Frau B. Gabriel aus der Klinik für bildgebende Diagnostik und Interventionsradiologie (Direktor: Dr. R. Braunschweig) der BG-Kliniken Bergmannstrost zur Verfügung gestellt.

Interessenkonflikt: Der korrespondierende Autor versichert, dass keine Verbindungen mit einer Firma, deren Produkt in dem Artikel genannt ist, oder einer Firma, die ein Konkurrenzprodukt vertreibt, bestehen.

\section{Literatur}

1. Bell WR, Simon TL (1982) Current status of pulmonary thromboembolic disease: pathophysiology, diagnosis, prevention, and treatment. Am Heart J 103: 239-262
2. Bounameaux H, Perrier A (1996) Role of D-dimers in the excusion of pulmonary embolism. Semin Respir Crit Care Med 17: 31-37

3. Fedullo PF, Tapson VF (2003) The evaluation of suspected pulmonary embolism. N Engl J Med 349: 1247-1256

4. Gallerani M, Manfredini R, Ricci L et al. (1992) Sudden death from pulmonary thromboembolism: chronobiological aspects. Eur Heart J 13: 661-665

5. Goldhaber SZ (1998) Pulmonary embolism. N Engl J Med 339: 93-104

6. Green RM, Meyer TJ, Dunn M et al. (1992) Pulmonary embolism in younger adults. Chest 101: 15071511

7. Heinrich F (1998) Organbezogene Venenthrombosen. In: Hach Wunderle V, Theiss W (Hrsg) Die Venenthrombose - Kontroversen. Springer, Berlin Heidelberg New York

8. Heintzen MP, Strauer BE (1999) Akutes Cor pulmonale bei Lungenarterienembolie. Internist 7: 710721

9. Heit JA, O'Fallon WM, Peterson TM et al. (2002) Relative impact of risk factors for deep vein thrombosis and pulmonary embolism. Arch Intern Med 162: $1245-1248$

10. Hofmann T, Meinertz T, Kasper W et al. (1992) Echokardiographie in der Diagnostik der Lungenembolie. Dtsch Med Wochenschr 117: 21-26

11. Kearon C (2003) Diagnosis of pulmonary embolism. Can Med Assoc J 21: 183-203

12. Konstantinides S, Hasenfuss G (2004) Akutes Cor pulmonale bei Lungenembolie - Entscheidender prognostischer Faktor und kritischer Parameter für die Auswahl der therapeutischen Strategie. Internist Online First (3. August)

13. Meany JFM, Weg JG, Chenevert TL et al. (1997) Diagnosis of pulmonary embolism with magnet resonance angiography. N Engl J Med 336: 1422-1427

14. Moses DC, Silver TM, Bookstein JJ (1974) The complementary roles of chest radiography, lung scanning and selective pulmonary angiography in the diagnosis of pulmonary embolism. Circulation 49: 179-188

15. Müller C, Kopka L, Funke M et al. (2001) Diagnostik der Lungenembolie und zugrunde liegender Venenthrombosen in der Mehrzeilen-Spiral-CT. Fortschr Röntgenstr Neuen Bildgeb Verfahr 173: 528535

16. Partsch H, Blättler W (1998) Leitlinien zur Thromboembolie-Prophylaxe. Phlebologie 27: 98-104

17. Pforte A (2004) Epidemiology, diagnosis, and therapy of pulmonary embolism. Eur J Med Res 9: 171179

18. Schoepf UJ, Kessler MA, Rieger C et al. (2001) Diagnostik der Lungenembolie mit der MehrschichtSpiral-CT. Radiologe 41: 248-255

19. Schümichen C (2002) Diagnostik der Lungenarterienembolie (2). Dtsch Med Wochenschr 8: 524-525

20. Seifried $E$, Heinrich F (2000) Klinik und Diagnostik. In: Seifried E, Heinrich F (Hrsg) Lungenembolie. Thieme, Stuttgart New York, S 23-48

21. Silverstein MD, Heit JA, Mohr DN et al. (1998) Trends in the incidence of deep vein thrombosis and pulmonary embolism: a 25-year populationbased study. Arch Intern Med 158: 585-593

22. Stein PD, Terrin Ml, Hales CA et al. (1991) Clinical, laboratory, roentgenographic, and electrocardiographic findings in patients with acute pulmonary embolism, and no pre-existing cardiac or pulmonary disease. Chest 100: 598-603 